\title{
An Adaptive Parameter Estimation in a BTV Regularized Image Super-Resolution Reconstruction
}

\author{
Mehdi MOFIDI ${ }^{1}$, Hassan HAJGHASSEM ${ }^{2}$, Ahmad AFIFI $^{1}$ \\ ${ }^{1}$ Department of Electrical Engineering, Malek Ashtar University of Technology, Tehran, Iran \\ ${ }^{2}$ Department of New Sciences \& Technologies, University of Tehran, Tehran, Iran \\ m.mofidi@aletaha.ac.ir
}

\begin{abstract}
Access to the fine spatial resolution has always been a hotspot in digital imaging. One way to improve resolution is to use signal post-processing techniques. In this study, an improved multi-frame image super-resolution (SR) algorithm is proposed. The objective function should be minimized consists of a data error term, a regularization term and a regularization parameter. Based on the bilateral-totalvariation (BTV) regularization, in the proposed method on one hand, the data error term incorporates frames with high accuracies in the reconstruction process, where an indicator weights each frame proportional to the frame error. On the other hand the regularization parameter is updated in each iteration based upon the Morozov's discrepancy principle. Iterative adjustment of the regularization parameter guarantees the SR solution to satisfy discrepancy principle. Visual evaluation and also quantitative measurements show that the performance of the proposed algorithm is better than of the several state-of-the-art methods.
\end{abstract}

Index Terms-image processing, image reconstruction, maximum a posteriori, spatial resolution, statistical analysis.

\section{INTRODUCTION}

The spatial resolution of an imaging system is always affected by hardware and physical constraints which lead to generate low-resolution (LR) images or sequence. However, in most applications high-resolution (HR) images are required and often desired. On the other hand, once an imaging system is manufactured, modifying the imaging hardware is not an available choice, thus highlighting a need for an image post-processing technique. One of these techniques is called super-resolution (SR) reconstruction which is a process that aims to fuse multiple LR images to compose a HR image. Generally, the relative sub-pixel motion between LR frames provides some redundant information which can be combined to produce a HR image. In SR literature, reconstruction-based algorithms are well developed in spatial domain. This can be due to their less sensitivity to errors and also more capability in incorporating prior knowledge about the HR image than the frequency domain [1]. The iterative back projection (IBP) [2], non-uniform interpolation [3], and the projection onto convex sets (POCS) [4] are such as non-statistical spatial domain approaches. Bayesian-based statistical algorithms, which are considered in this paper, consist of maximum likelihood (ML), maximum a posteriori (MAP), hybrid ML/MAP/POCS, variational Bayesian [5-9] and so forth. Since the SR reconstruction is an ill-posed problem, Bayesian regularized algorithms have become an appealing research topic in this area. This can be due to their strong statistical inference, robustness against the errors, and employment of priors as the regularization terms. A rich class of regularization-based functions is the MAP estimator. Generally, the MAP estimation can be formulated as the solution of a two-term optimization problem of the form [10]:

$$
\min _{x_{H}}\left[C\left(x_{H}, x_{L}\right)+\lambda R\left(x_{H}\right)\right]
$$

where $C($.$) is the data error term, measuring lack of$ similarity to the data $x_{L}, R($.$) is a measure of$ smoothness/roughness of solution which called regularization function and $\lambda>0$ is the regularization parameter, attributing $C$ and $R$. There are two key points in successfully solving the MAP-based super-resolution problem: 1) specification of the methods entail making choice of $C$ and $R$, and 2) estimation of the regularization parameter $\lambda$ that balance the relative weights between $C$ and $R$. Estimators include Lorentzian, Huber, Tukeys' Biweight, $L_{1}$ and $L_{2}$ norms have been used as data error term $C$, in several works [10-13]. The $L_{1}$ norm has a good performance of preserving edges for the speckle noise model and misregistration errors, whereas the $L_{2}$ norm for Gaussian noise model can well keep the smoothness of the image. This motivates everyone to find a way which merges both advantages of $L_{2}$ and $L_{1}$ norms. The success of other above-mentioned estimators is highly dependent on accurate selection of their threshold values. As a choice of regularization term $R$, Tikhonov method [14] is one of the most classical regularization terms which introduces smoothness constraints in reconstructed image, but it loses sharpness in the HR image. Total variation (TV) [15] is another regularizer which overcomes the shortcoming of Tikhonov method and is widely used in image restoration tasks. A progressive regularization function presented in [16] based on a bilateral total variance (BTV) as a modification of TV method. We will also use BTV as regularization term, in our formulation.

As one of the objectives, we focus our attention on the second mentioned essential problem, finding the regularization parameter $\lambda$. However the wise choice of $\lambda$ is a delicate object: if $\lambda$ is too large, the super-resolved image is oversmoothed, and conversely, if $\lambda$ is too small, the noise will not be effectively suppressed. In many works the regularization parameter is determined manually by trial- 
and-error. However, this procedure can be time-consuming and chanceful. To avoid these drawbacks, up to now, several adaptive selection strategies have been proposed. For example, the generalized cross-validation (GCV) method [17], the L-curve method [18], the U-curve method [19], the variational Bayesian framework [9], and the discrepancy principle [20]. The GCV method is based on a-priori knowledge of the input errors in LR frames [21] and it can be used when the regularization term is a quadratic function. It is well known that the computational cost of GCV is high [19]. The L-curve criterion is implemented by the plot of the norm of regularization term versus the norm of the corresponding data error term. Generally, the L-curve has a "vertical" part and a "horizontal" part. The optimum regularization parameter is located in the L-shaped corner of the L-curve. Sometimes, finding the exact corner of the curve is difficult. Moreover, to do so, one should solve (1) many times for different $\lambda$ 's. Another practical method is the U-curve criterion which has a similar procedure to Lcurve method. The U-curve provides a truncated interval where the optimal $\lambda$ locates. Therefore, as the main difference, U-curve has a reduced computational cost than the L-curve method. The variational Bayesian framework can provide good solutions, but since it is dependent on some attached parameters, it is not fully adaptive. In image restoration field with a single LR frame, based on the primal dual approach and discrepancy principle, an efficient TVregularized restoration problem is handled in [22]. Another TV-based image restoration work using variable splitting technique is presented in [23] based on discrepancy principle. In this paper, in order to select the regularization parameter we use the Morozov's discrepancy principle, which less attention has given to it in super-resolution literature. This method chooses $\lambda$ by adjusting the discrepancy between observed and expected images to some upper bounds. We should emphasize on major differences between our method and other methods using the discrepancy principle. Based upon our knowledge, most of the presented works are in image restoration field, not SR reconstruction, where only one LR frame is employed. These methods are based on TV regularization, suffer from their nondifferentiability. In this case some numerical methods including variable splitting algorithms [24] and primal dual model [22] should be employed which require to introduce some auxiliary variables [23]. Therefore, they are more complex than our algorithm, where BTV regularization is used and no variable is introduced in minimization problem. In addition, BTV method could preserve edges and suppress noise better than TV method [25]. Our objectives in this paper are twofold. First, we suggest a modified data error term which assigns proper weight to each frame based upon the frame error. In this strategy, frames with higher accuracies will have more contribution in SR reconstruction. Second, as a more adaptive solution, unlike the existing optimized methods that focus on problems with a fixed $\lambda$, we propose a discrepancy based regularization parameter selection in an adaptive way. The remainder of this paper is structured as follows: the observation model for image acquisition is provided in section II. Section III describes the proposed reconstruction model. Experimental results and discussion are given out in Section IV. Finally, a conclusion is drawn in Section V.

\section{IMAGING OBSERVATION MODEL}

The degradation procedure in an imaging system can relate HR and LR frames through a mathematical forward model. Assuming the degradation involves downsampling, Gaussian blurring, warping and additive noise, the model can be expressed linearly as:

$$
\underline{X}_{L}^{k}=D H_{k} F_{k} \underline{X}_{H}+E_{k}
$$

where $\underline{X}_{L}^{k}$ denotes to the multiple LR images that are used to generate a single HR image $\left(\underline{X}_{H}\right)$ and $k$ is the number of LR images $(k=1,2, \ldots, K)$. In above forward model warping operator $\left(F_{k}\right)$ encodes the motion information of the $k$-th LR frame to $\underline{X}_{H}$. Blurring effect is realized by applying the blurring $\operatorname{kernel}\left(H_{k}\right)$. The warped and blurred images are then downsampled by decimation operator $D . \underline{E}_{k}$ is the noise term that finally adds to the process. The matrices $\underline{X}_{L}^{k}, \quad D, H_{k}, F_{k}, \underline{X}_{H}$ and $\underline{E}_{k}$ are of sizes $M N \times 1$, $M N \times r^{2} M N, r^{2} M N \times r^{2} M N, r^{2} M N \times r^{2} M N, r^{2} M N \times 1$ and $M N \times 1$, respectively, where $r$ is the integer-valued interpolation factor.

\section{Proposed Model For SR Reconstruction BASED ON ADAPTIVE TERMS}

\section{A. Data error term}

Generally, in SR image reconstruction, a sequence of LR images is fused to produce a higher resolution image. In doing so, accurate motion estimation, precise mapping of the LR pixels on to the HR frame, and blurring kernel estimation/assumption is essential to the success of any SR algorithm. Moreover, noise model, noise level, and existence of outliers in the LR frames are as influential factors on the final result. Therefore, a robust estimation method that is not such sensitive to errors may produce more stable results. A pervasive family of estimators is the ML estimators. Based upon the ML concept, the estimation of $\hat{X}_{H}$ is done through the following minimization expression [13]:

$$
\hat{X}_{H}=\arg \min _{X_{H}}\left[\sum_{k=1}^{K} \rho\left(D H_{k} F_{k} \underline{X}_{H}-\underline{X}_{L}^{k}\right)\right]
$$

where $\rho\left(D H_{k} F_{k} \underline{X}_{H}-\underline{X}_{L}^{k}\right)$ is the data error term, measuring the "residual" between the observation and the estimation models. Defining $x$ as the residual error, mathematically, $\rho(x)$ could be an even positive function with a unique minimizer at $x=0$ Derivative of $\rho(x)$ with respect to $X_{H}$, denoted $\varphi(x)=\left(\partial / \partial X_{H}\right)(\rho(x))$ is called the influence function, and can be used to define the bias that a particular observation has on the solution [26]. $\rho(x)$ should be chosen according to the distribution of the residual errors. Considering this, some proper choices for $\rho(x)$ are the $L_{1}$, $L_{2}$, and $L_{p}$ norms $(0<p \leq 1)$. The error function of $L_{1}$ norm is $\rho(x)=|x|$, and $\varphi(x)=\operatorname{sign}(x)$. Due to the constancy 
and bounded value of influence function ( -1 or 1 ), the $L_{1}$ norm doesn't distinguish between large errors corresponding by outliers or small errors caused by additive noise [27]. For $L_{2}$ norm, $\rho(x)=0.5 x^{2}$ and therefore $\varphi(x)=x$ that obviously increases linearly and without bound with respect to $x$. This linear proportionality to residual errors causes the poor performance of $L_{2}$ norm in the presence of outliers or large errors [16]. As a result, $L_{1}$ norm is not sensitive to large errors but when the residual errors are relatively small, $L_{2}$ norm has a better performance than the $L_{1}$ norm. Moreover, $L_{1}$ does not have an analytical solution, but $L_{2}$ norm does. In such a situation, a model with a hybrid effect that has the advantages of both $L_{1}$ and $L_{2}$ norm terms can perform better than $L_{1}$ or $L_{2}$ norms. Various ML-estimators such as Lorentzian, Huber and Tukeys' Biweight are proposed in [11-13]. All of these estimators have a combinational behavior of both $L_{1}$ and $L_{2}$ norms which uses a threshold to define the behavior of error norm adaptively. In other words, for values smaller than a threshold the function follows $L_{2}$ norm and for values larger than threshold they perform like $L_{1}$ norm. Therefore, an adaptive threshold selection for these M-estimators has a key role in the success of superresolution reconstruction. The Half-quadratic estimation is one of the adaptive error functions with above mentioned properties which is proposed in [27]:

$$
\rho(x, a)=a \sqrt{a^{2}+x^{2}}-a^{2}
$$

where $a$ is a positive number. $\rho(x, a)$ is convex and twice continuously differentiable which guarantees a unique solution of the optimization problem. The influence function of $\rho(x, a)$ is defined as:

$$
\phi(x, a)=a x / \sqrt{a^{2}+x^{2}}
$$

with a fixed $a$, when $x$ is relatively small, $\rho(x, a) \approx x^{2} / 2$ and the function behaves like $L_{2}$ norm. On the other hand, when $x$ is large enough, $\rho(x, a) \approx a|x|-a^{2}$ and the function takes the shape of $L_{1}$ norm. The selection of $L_{1}$ norm or $L_{2}$ norm and the transition between them can be controlled by modifying the parameter $a$. In other words, for large values of $x$ (large errors), $a$ should be small and conversely, for small values of $x$ (small errors), $a$ should be large. Accordingly, $\rho(x, a)$ is an adaptive robust norm. Substituting (4) in (3), after one step manipulation, one can result in:

$$
\hat{X}_{H}=\arg \min _{X_{H}}\left[\sum_{k=1}^{K} a_{k} \sqrt{a_{k}^{2}+\left(D H_{k} F_{k} \underline{X}_{H}-\underline{X}_{L}^{k}\right)^{2}}-a_{k}^{2}\right]
$$

where $a_{k}$ is the threshold parameter for the $k$-th frame. Each of the LR frames can have its own $a_{k}$, due to the different accuracy of frames in registration step. In other words, $a_{k}$ acts as a frame indicator which is inversely proportional to the frame errors. It is clear that, more erroneous frames should have less participation in the final HR result. A method for choosing $a_{k}$ is proposed in [27], but it has a relative high computational cost. Herein, we introduce a new formulation for $a_{k}$, while simplicity, provides acceptable results. Considering $X_{H}(0)$ as the first HR estimation, $a_{k}$ can be written as:

$$
a_{k}=\sqrt{E_{k_{\max }}-E_{k_{\min }}} / E_{k}^{2}
$$

where $E_{k}=\left\|D H_{k} F_{k} \underline{X}_{H}(0)-\underline{X}_{L}^{k}\right\|_{2} / U$ is the k-th frame error term. $E_{k_{\max }}$ and $E_{k_{\min }}$ are the maximum and minimum of all $E_{k}$ values, respectively. In this case, by increasing $E_{k}, \quad a_{k}$ will decrease and the frame weight in SR reconstruction will reduce. In our experiment, $X_{H}(0)$ is the up-scaled version of a random LR frame by bilinear interpolation.

\section{B. Regularization using BTV function}

Direct inversion of matrix multiplication $D_{k} F_{k}$ in (2), is not usually a viable approach due to its large dimensions and the ill-posedness of the problem [28]. In this case, regularization stabilizes the inverse problem appropriately, while providing a reasonable solution to the original problem. There are, however, different possibilities for the regularization term. One of the referenced functions called bilateral total variation (BTV) is proposed in [16] as a modification of TV method:

$$
R_{B T V}\left(X_{H}\right)=\sum_{l=-P}^{P} \sum_{m=-P}^{P} \alpha^{|m|+|l|}\left\|\underline{X}_{H}-S_{x}^{l} S_{y}^{m} \underline{X}_{H}\right\|_{1}
$$

where $S_{x}^{l}$ and $S_{y}^{m}$ shift $X_{H}$ by $l$ and $m$ pixels in the $x$ and $y$ directions, respectively. The weight $0<\alpha<1$ imposes a spatial decaying effect to regularization terms and $P$ defines the size of the regularization kernel. BTV method is computationally efficient, and it tends to preserve edges. By substituting data error term (6) and regularization term (8) in (1), the desired solution $X_{H}$ can be obtained efficiently using iterative methods like steepest descent (SD) algorithm through $X_{H}^{i+1}=X_{H}^{i}-\beta \nabla X_{H}^{i}$. In this equation, $i$ refer to the iteration number, $\beta$ is the step size in the negative direction of the gradient and $\nabla$ is the derivative operator. Accordingly, optimal $X_{H}$ can be found, as follows:

$$
\begin{aligned}
& X_{H}^{i+1}=X_{H}^{i}-\beta\left(\sum_{k=1}^{K}\left(D H_{k} F_{k}\right)^{T} A_{k}\left(D H_{k} F_{k} \underline{X}_{H}^{i}-\underline{X}_{L}^{k}\right)\right. \\
& \left.+\lambda \sum_{l=-P}^{P} \sum_{m=-P}^{P} \alpha^{|l|+|m|}\left[I-S_{x}^{-l} S_{y}^{-m}\right] \operatorname{sign}\left(\underline{X}_{H}^{i}-S_{x}^{l} S_{y}^{m} \underline{X}_{H}^{i}\right)\right)
\end{aligned}
$$

where $S_{x}^{-l}$ and $S_{y}^{-m}$ are the transpose of $S_{y}^{l}$ and $S_{y}^{m}$, respectively and $A_{k}=a_{k} / \sqrt{a_{k}^{2}+\left(D H_{k} F_{k} \underline{X}_{H}^{i}-\underline{X}_{L}^{k}\right)^{2}}$. Regularization parameter $\lambda$ has a vital role in the optimization process. In the following, we propose a strategy to choose regularization parameter adaptively, in each iteration of optimization.

\section{Adaptive method for regularization parameter selection}

One of the main operational problems in (1) (as well as in (9)) is how to choose the regularization parameter $\lambda$. There are different posteriori strategies for choosing $\lambda$ in the literature. In this regard, we will use the Morozov's 
discrepancy principle which has been studied less in SR reconstruction. One can assume that the discrepancy between the observed and the estimated frames is defined as:

$$
e^{i+1}(\lambda)=\sum_{k=1}^{K}\left(X_{L}^{k}-D H_{k} F_{k} X_{H}^{i+1}\right)
$$

According to this principle, a good solution of the image restoration problem based on a single frame should lie in $\left\|X_{L}-D H F X_{H}\right\|_{2}^{2} \leq m n \sigma^{2}$ [29], where $\sigma^{2}$ is the variance of the noise. In the case of image super-resolution problem, where $K$ is the number of LR frames, we can express:

$$
\left\|e^{i+1}(\lambda)\right\|_{2}^{2} \leq K m n \sigma^{2}
$$

where $\left\|e^{i+1}(\lambda)\right\|_{2}^{2}$ is a positive and decreasing convex function of $\lambda$. It means that if the noise power is known, (11) provides a bound on discrepancy $e^{i+1}(\lambda)$. So in particular, parameter $\lambda^{i+1}$ in the $(i+1)$-th iteration could be chosen by requiring $\left\|e^{i+1}(\lambda)\right\|_{2}^{2}=K m n \sigma^{2}$ By substituting $X_{H}^{i+1}$ from (9) in (10) the following formula can be written:

$$
e^{i+1}(\lambda)=\sum_{k=1}^{K}\left(X_{L}^{k}-D H_{k} F_{k}\left(X_{H}^{i}-\beta\left(G_{1}+\lambda G_{2}\right)\right)\right)
$$

where $G_{1}$ and $G_{2}$ are the derivatives of data error term and regularization term, respectively:

$$
\begin{gathered}
G_{1}=\left(\sum_{k=1}^{K}\left(\left(D H_{k} F_{k}\right)^{T}\left(a_{k} / \sqrt{a_{k}^{2}+\left(D H_{k} F_{k} \underline{X}_{H}^{i}-\underline{X}_{L}^{k}\right)^{2}}\right)\left(D H_{k} F_{k} \underline{X}_{H}^{i}-\underline{X}_{L}^{k}\right)\right)\right) \\
G_{2}=\left(\sum_{l=-P m=-P}^{P} \sum^{P}\left(\alpha|||+| m \mid\left[I-S_{x}^{-l} S_{y}^{-m}\right] \operatorname{sign}\left(\underline{X}_{H}^{i}-S_{x}^{l} S_{y}^{m} \underline{X}_{H}^{i}\right)\right)\right)
\end{gathered}
$$

By separating the terms involving parameter $\lambda$ from the others in (12), we will have:

$$
e^{i+1}(\lambda)=A+\lambda B
$$

where:

$$
\begin{gathered}
A=\sum_{k=1}^{K}\left(X_{L}^{k}-D H_{k} F_{k}\left(X_{H}^{i}-\beta G_{1}\right)\right) \\
B=\sum_{k=1}^{K}\left(D H_{k} F_{k} \beta G_{2}\right)
\end{gathered}
$$

Since $\left\|e^{i+1}(\lambda)\right\|_{2}^{2}=\left(e^{i+1}(\lambda)\right)^{T}\left(e^{i+1}(\lambda)\right)$, by substituting (15) in this equation, after some manipulation, one can express:

$$
\left\|e^{i+1}(\lambda)\right\|_{2}^{2}=A^{T} A+\lambda\left(A^{T} B+B^{T} A\right)+\lambda^{2} B^{T} B
$$

Finally by equating (18) to $K m n \sigma^{2}, \lambda$ can be calculated in each iteration. As can be seen, this calculation requires solving a nonlinear equation in order to obtain the value of the regularization parameter $\lambda$. We use the Gauss-Newton algorithm to find $\lambda$ iteratively. The resulting algorithm for SR reconstruction is summarized in Fig.1.

\section{EXPERIMENTAL RESULTS}

To evaluate the performance of the proposed SR algorithm, simulation experiments are carried out in MATLAB R2009a environment on a laptop computer with Intel core i5 processor running at $2.53 \mathrm{GHz}$ clock with an $8 \mathrm{~GB}$ internal RAM memory.

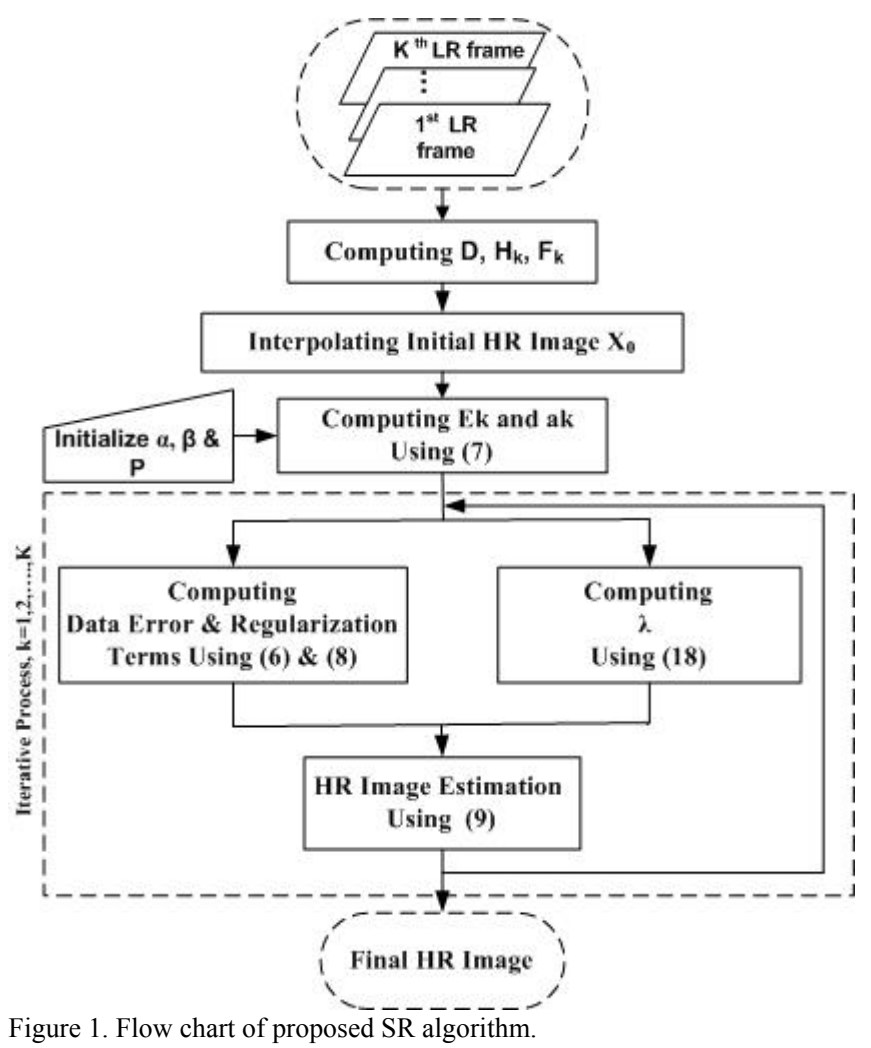

The experiments consist of two parts. In the first part (Subsection A), the experiments come to test the performance of proposed algorithm on some synthetic images.

The LR images in this part are acquired from a singleframe HR image by a degradation procedure which is described in the same section. In the second part (Subsection B), we turn to real images in order to test the effectiveness of the SR model. In both subsections, the performance of data error term is investigated first. To this end, at first, regularization term (BTV) is kept constant and $\lambda$ is selected manually to have a fair comparison with other two methods. By fixing the regularization term, we justify the effectiveness of our proposed data error term, by comparing it with the $L_{1}$ norm [16] "denoted as $L_{1}+\mathrm{BTV}$ " and $L_{2}$ norm [30] "denoted as $L_{2}+B T V "$ ". Furthermore, the results of proposed method with manual selection of regularization parameter and Half-quadratic method [30] are presented. In the next experiment, adaptive selection of $\lambda$ using the proposed method is compared with GCV [17] and L-Curve [18] methods which are applied for automatic selection of regularizing parameter.

\section{A. Synthetical experiments}

In this section, two original Remote Sensing images of sizes $360 \times 360$ pixels are chosen which are depicted in Fig. 2(b) and Fig. 3(b). Four LR Remote Sensing images are generated by shifting Fig. 2(b) and Fig. 3(b) horizontally and vertically, blurring them with a zero-mean $5 \times 5$ Gaussian kernel with standard deviation of 1 , and downsampling them by factor of 2. Figs. 2(a) and 3(a) show one of the resulted LR frames (of size $180 \times 180$ ) in each Remote sensing images. It should be noted that the SR model parameters such as motion vectors $\left(F_{k}\right)$, blurring kernels $\left(H_{k}\right)$, and additive noise $\left(E_{k}\right)$ are unknown and 
needed to be estimated. In the experiments, an efficient phased based image matching method [31] is employed for motion estimation. After this step, $H_{k}$ is assumed to be a $5 \times 5$ Gaussian kernel with standard deviation equal to 1 . We used the method described in [32] to estimate the additive noise variance. The values of fixed parameters in SR model are as follows: $\alpha=0.5$ and $P=2$. For a fair evaluation of performance, the parameters of other existing algorithms are chosen in several trials to produce most appealing results. To facilitate a better comparison, a region is cropped and shown at the bottom-right of each image. Fig. 2(c) and Fig. 3 (c) show the result of using $L_{2}+\mathrm{BTV}$ algorithm. From this result, it is clear that $L_{2}$ norm suffers from obvious noise and artifacts. Observation from Fig. 2(d) and Fig. 3(d) suggest that the use of $L_{1}+\mathrm{BTV}$ term, in spite of suppressing the noise and artifacts, reduces the image quality significantly. This might be because $L_{2}$ norm differentiates between large errors and small errors, and assigns a distinct weight to each one. Whereas $L_{1}$ assigns identical weights to all errors and this mode creates it more undesirable compared to $L_{2}$ norm. As shown in Fig. 2(e) and Fig. 3(e), unlike $L_{2}+\mathrm{BTV}$ and $L_{1}+\mathrm{BTV}$ cases, the Half-quadratic method proposed in [27] has a better visual results but it lacks sharp edges and fine details.

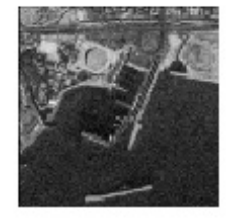

(a)

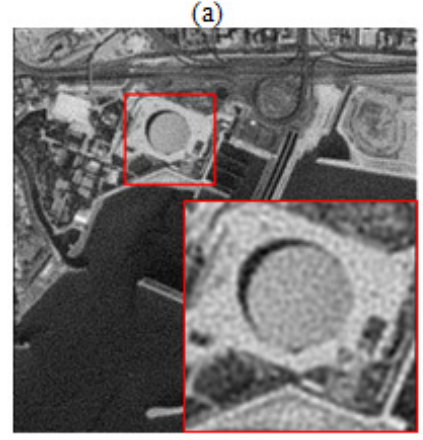

(c)

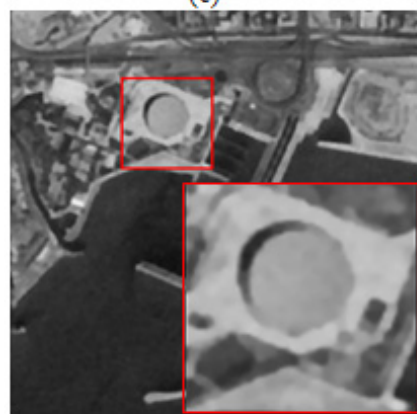

(e)

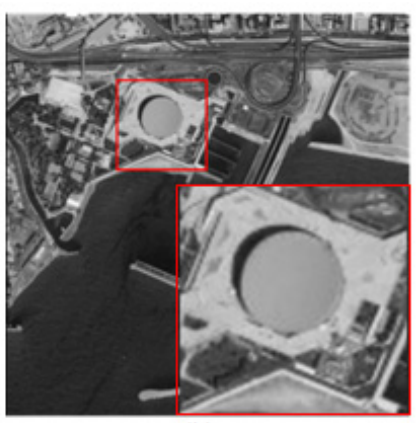

(b)

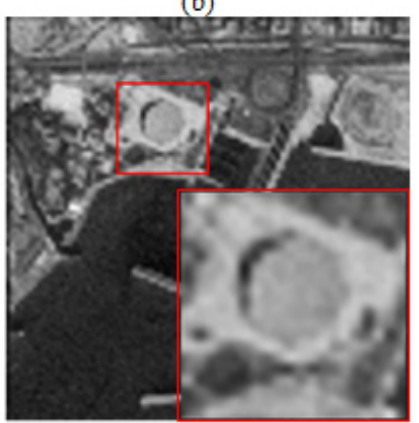

(d)

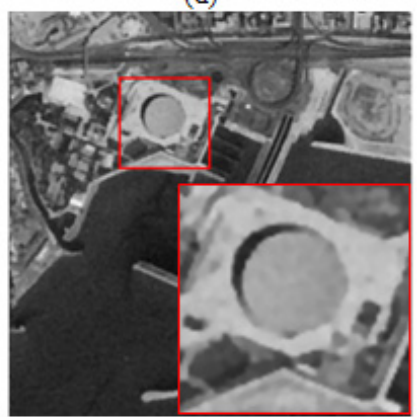

(f)
Figure 2. Reconstruction results of Remote Sensing1 sequence. (a) One of LR-Images, (b) original HR-Image, (c) result of $L_{2}+\mathrm{BTV}$, (d) result of $L_{1}+\mathrm{BTV}$, (e) result of Half-quadratic method, and (f) result of proposed method with manual selection of $\lambda$.
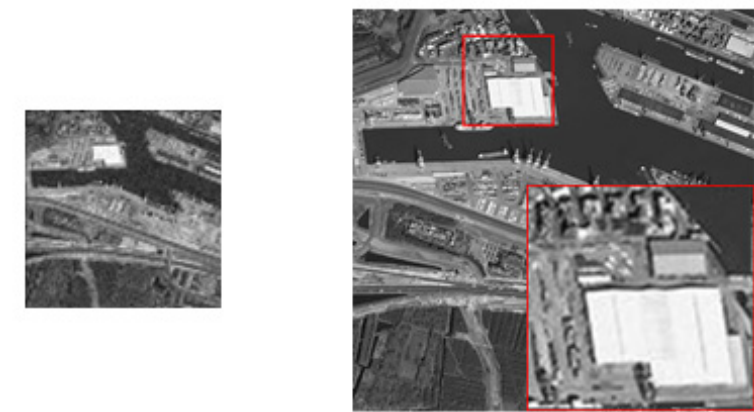

(a)

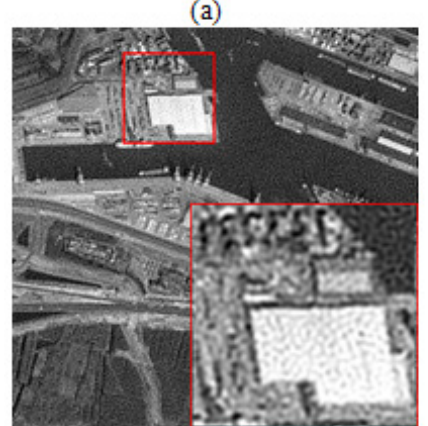

(c)

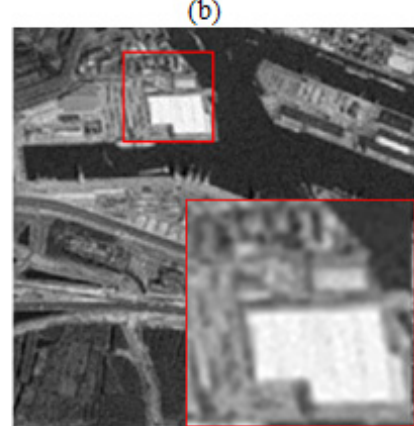

(d)

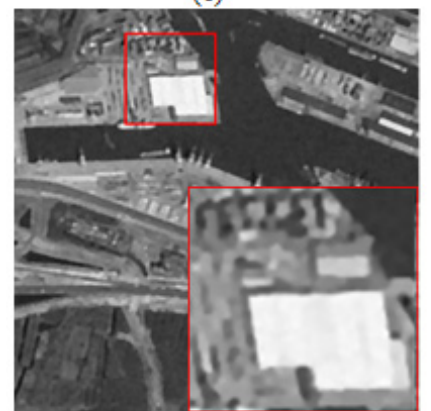

(e)

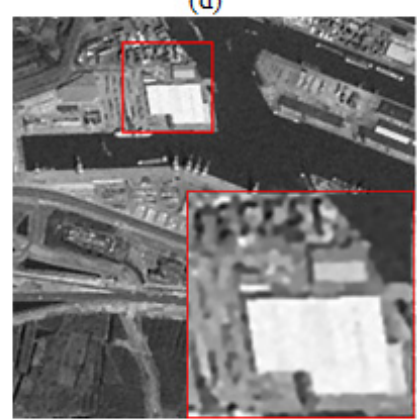

(f)

Figure 3. Reconstruction results of Remote Sensing2 sequence. (a) One of LR-Images, (b) original HR-Image, (c) result of L2+BTV, (d) result of L1+BTV, (e)result of Half-quadratic method, and (f) result of proposed method with manual selection of $\lambda$

Fig. 2(f) and Fig. 3(f) show the reconstruction result of proposed method under the manual selection of regularization term. This may, in part, be caused by adaptive data error term which assigns smaller weights $a_{k}$ to the LR frames that are corrupted by more errors.

In this subsection, we have judged the quality of reconstructed image quantitatively by well-known peak signal-to-noise ratio (PSNR) and structural similarity (SSIM) [33-34] indices. It should be mentioned that in this part, regularization parameter is selected manually for better comparison of data error terms.

\begin{tabular}{cccc}
\multicolumn{2}{c}{ TABLE I. PSNR AND SSIM VALUES FOR FIGURE 2 AND FIGURE 3} \\
\hline Image & Method & PSNR(dB) & SSIM \\
\hline \multirow{4}{*}{ Remote } & $L_{2}+$ BTV & 26.1952 & 0.5478 \\
Sensing1 & $L_{1}+$ BTV & 21.6908 & 0.577 \\
& GCV & 22.4791 & 0.6115 \\
& L-curve & 24.4821 & 0.6881 \\
& Half_quadratic & 25.3732 & 0.7146 \\
& Proposed_manual & 26.5292 & 0.7304 \\
Remote & Proposed_adaptive & $\mathbf{2 6 . 9 4 6 4}$ & $\mathbf{0 . 7 6 7 1}$ \\
\hline & $L_{2}+$ BTV & 23.0101 & 0.6008 \\
Sensing2 & $L_{1}+$ BTV & 21.3949 & 0.6150 \\
& GCV & 21.5683 & 0.6002 \\
& L-curve & 21.6696 & 0.6227 \\
& Half_quadratic & 22.2084 & 0.6463 \\
& Proposed_manual & 23.1147 & 0.6811 \\
& Proposed_adaptive & $\mathbf{2 3 . 4 6 0 6}$ & $\mathbf{0 . 7 0 4 7}$ \\
\hline
\end{tabular}



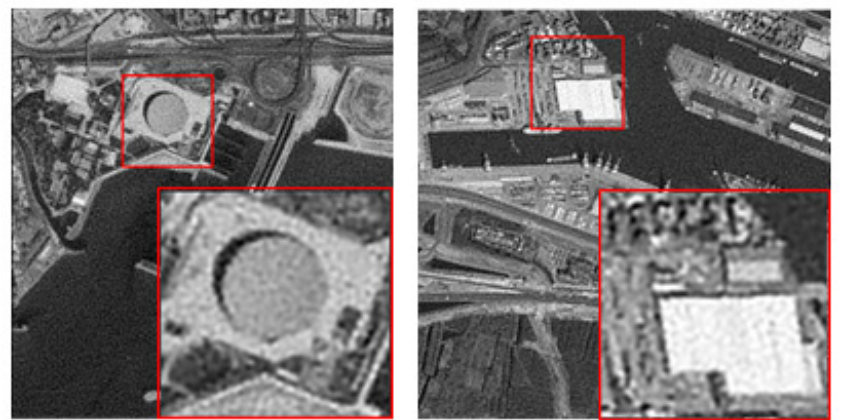

(a)
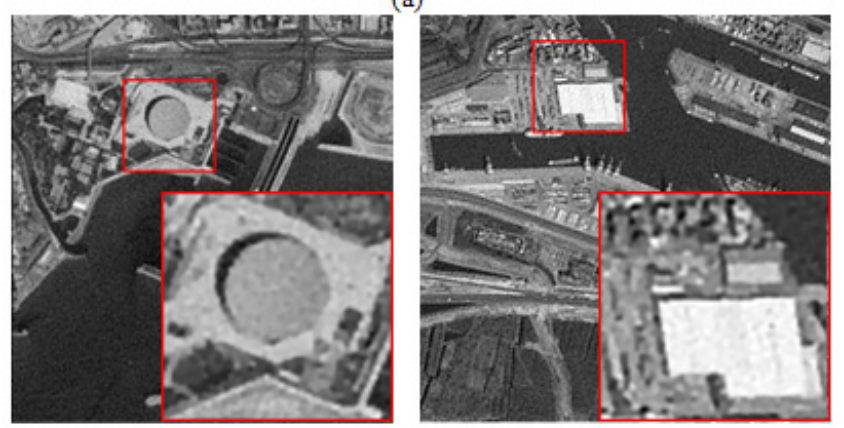

(b)
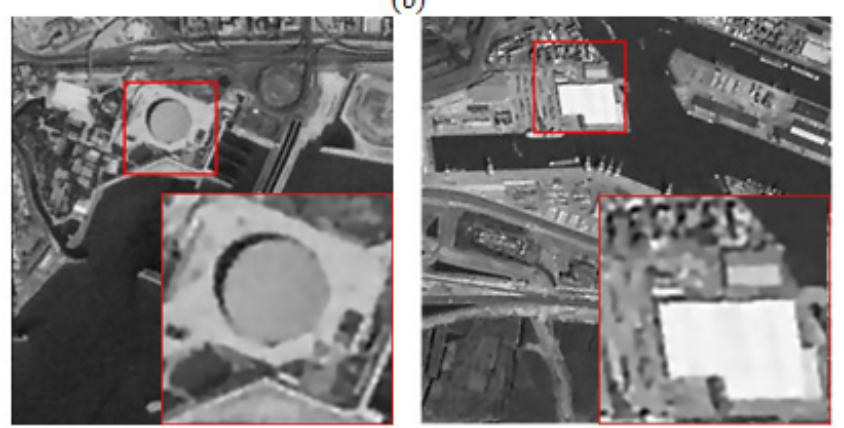

(c)

Figure 4. Reconstruction results of Remote Sensing1 and Remote Sensing2 sequences. (a) result of GCV, (b) result of L-curve, and (c) result of proposed method with adaptive selection of $\lambda$.

However, the results of the proposed method by automatically selecting $\lambda$ are also reported in Table I. Evidently, from Table I, we can see that our proposed method outperforms other methods and achieves the highest PSNR and SSIM results.

Fig. 4 compares GCV and L-curve algorithms with proposed method, which all of them are used for automatic selection of $\lambda$. As can be seen, GCV method adds a considerable noise to the resulted image. Noise of L-curve is less than the noise added by GCV but still quality of the image is poor. Finally, based on proposed method in Fig. 4, noise is eliminated considerably, however edges are sharper.

For better quantitative comparison of data error terms, the effect of varying regularization parameter $\lambda$ on SSIM values is plotted for different methods in Remote sensing1 and Remote sensing 2 images. As can be seen in Figs. 5(a) and 5(b), the proposed method has allocated a higher numerical result (SSIM) for all values of $\lambda$. Half-quadratic method also shows an appropriate behavior, but it ranks second in terms of performance. In general, $L_{1}+\mathrm{BTV}$ and $L_{2}+\mathrm{BTV}$ methods are ranked $3^{\text {rd }}$ and $4^{\text {th }}$, respectively.

Fig. 6 shows performance of the mentioned methods for automatic selection of $\lambda$ based on number of iterations and SSIM value. Less iterations for achieving a higher SSIM in proposed method is a key point which indicates simplicity and better performance of this algorithm.

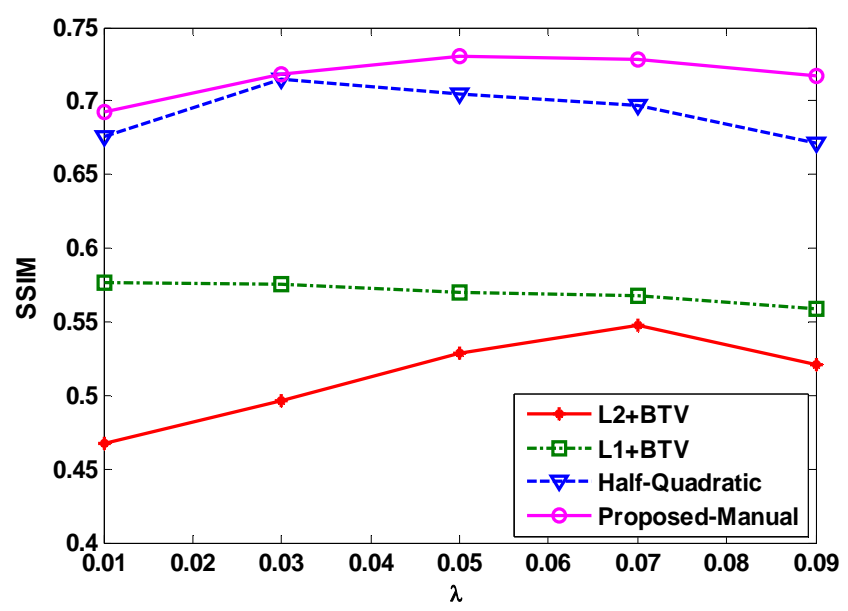

(a)

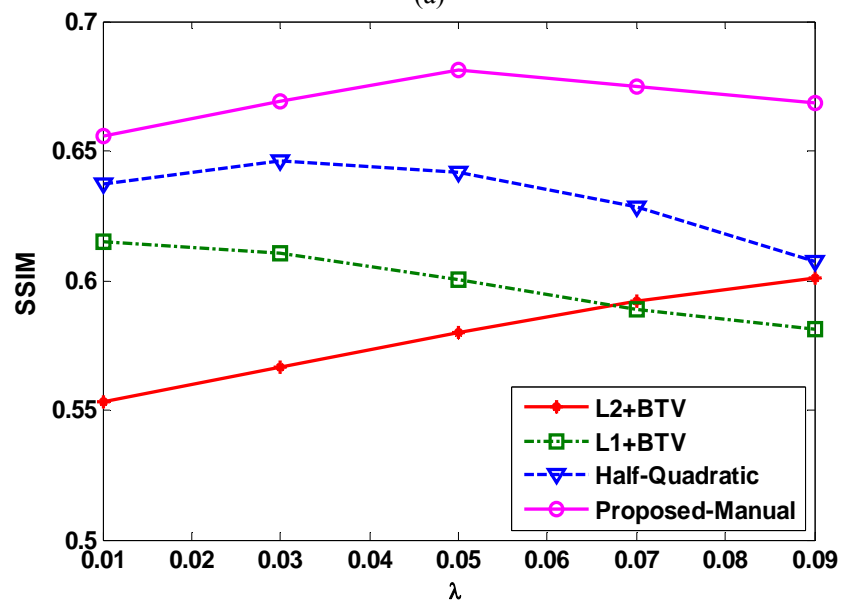

(b)

Figure 5. SSIM versus regularization parameter $\lambda$, (a) result for Remote Sensing1, (b) result for Remote Sensing2.

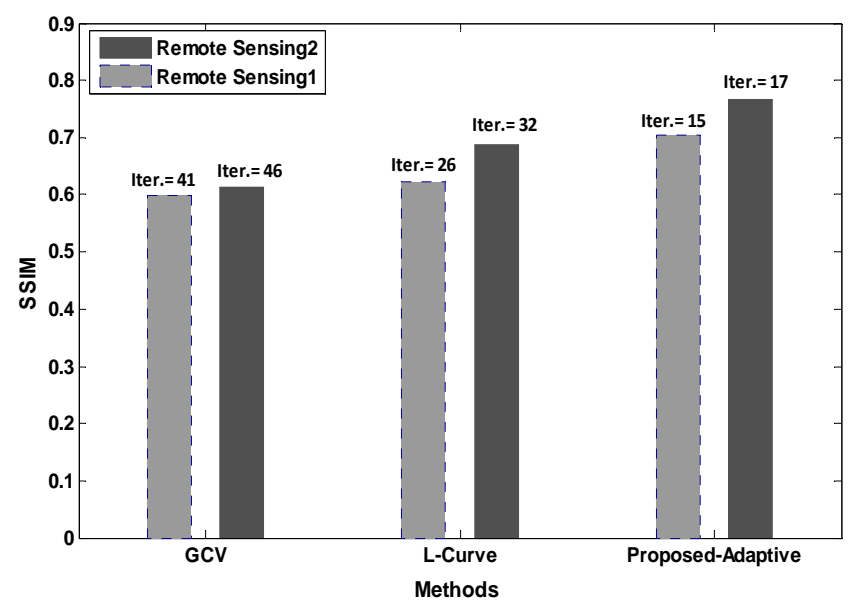

Figure 6. Performance of different regularization parameter selection methods in terms of iteration number and SSIM value.

For example, it can be seen that for obtaining an identical SSIM, the number of iterations in proposed method is $1 / 3$ of GCV method.

\section{B. Real-world experiments}

For a better comparison, proposed method is also applied to the real sequence "text". This sequence consists of 30 images (of size $57 \times 49$ pixels). Considering zoom factor of 4 , after SR reconstruction resolution of images reach to $228 \times 196$ pixels. The motion vectors $\left(F_{k}\right)$, blurring kernels $\left(H_{k}\right)$, and additive noise $\left(E_{k}\right)$ in SR model are unknown 
which are estimated in the same way of the previous section.

In the real-world experiment, since no ground truth HR image is available, the reconstruction results can be assessed by a no-reference image quality measure. Metric-Q and cumulative probability of blur detection (CPBD) are two image quality measures proposed in [35] and [36], respectively. Metric-Q employs the singular value decomposition of the local image gradients to evaluate the sharpness and contrast of reconstructed image. According to the Metric-Q, the higher the value is, the better the sharpness and the contrast will be. CPBD measure mainly assesses the image sharpness. CPBD ranges in [0-1] and the higher the value is, the better the sharpness will be.

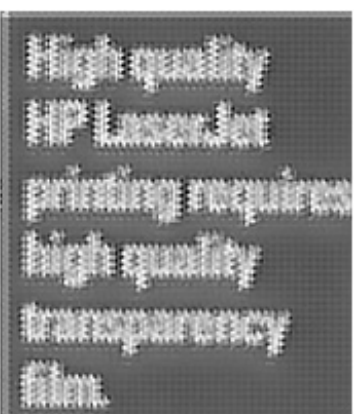

(a)

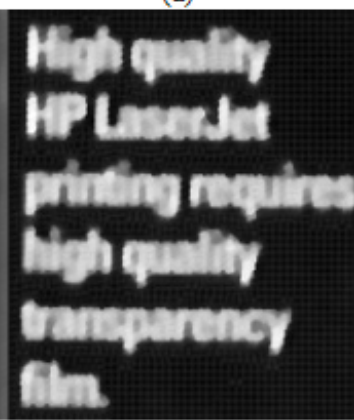

(c)

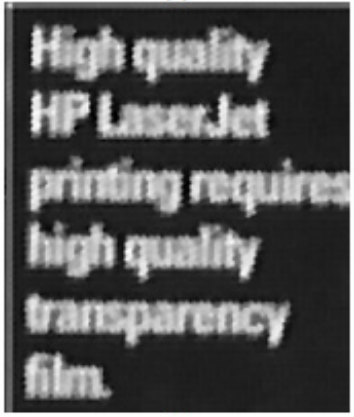

(e)

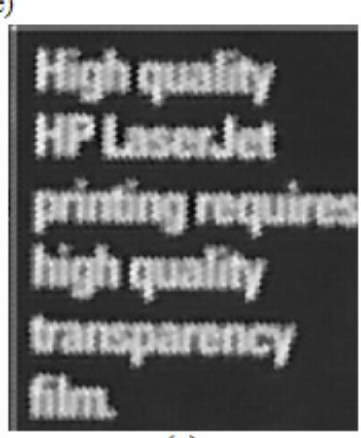

(g)

Figure 7. Reconstruction results of real sequence text. (a) result of $L_{2}+\mathrm{BTV}$, (b) result of $L_{1}+\mathrm{BTV}$, (c) result of Half-quadratic method, (d) result of GCV, (e) result of L-curve, (f) result of proposed method with manual selection of $\lambda$, and $(\mathrm{g})$ result of proposed method with adaptive selection of $\lambda$.

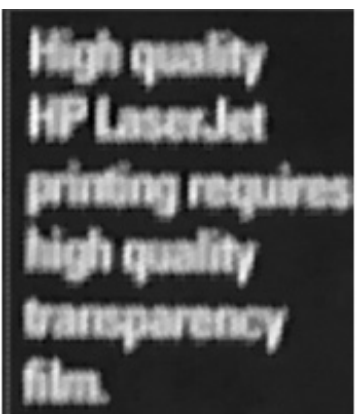

(b)

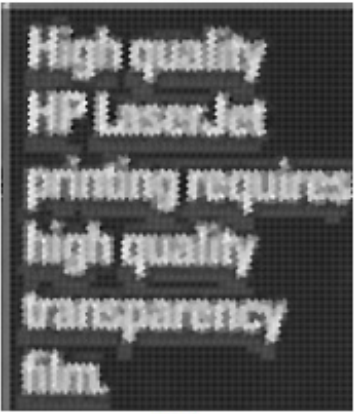

(d)

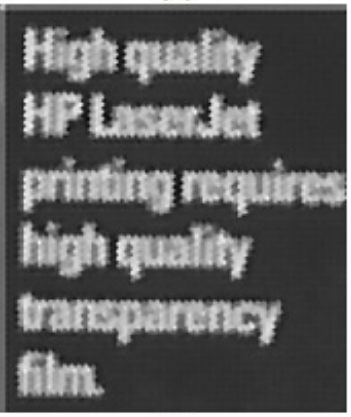

(f)

\begin{tabular}{cccc}
\hline Image & Method & CPBD & Metric_Q \\
\hline \multirow{6}{*}{ text } & $L_{2}+$ BTV & 0.4517 & 18.5375 \\
& $L_{1}+$ BTV & 0.4351 & 19.5519 \\
& GCV & 0.4493 & 19.0984 \\
& L-curve & 0.4548 & 19.8322 \\
& Half_quadratic & 0.4273 & 19.6978 \\
& Proposed_manual & 0.4554 & 19.9216 \\
& Proposed_adaptive & $\mathbf{0 . 4 6 9 5}$ & $\mathbf{2 0 . 0 8 9 3}$ \\
\hline
\end{tabular}

As can be seen in Fig. 7 (a), result of $L_{2}+$ BTV norm is an output with boosted effect of noise which can be due to its non-robust behavior against large errors. Although in Fig. 7(b), $L_{1}+$ BTV has a successful noise removal, but the image is oversmoothed and the details are destroyed. Result obtained from Half-quadratic method Fig. 7 (c) is still blurred. Due to Fig. 7 (d), applying GCV method for automatic selection of $\lambda$ creates considerable artifacts and noise. Fig. 7 (e) is obtained from L-curve which gives better results than GCV method.

Finally, In Figs. 7 (f) and (g) which are obtained from proposed method with manual and automatic $\lambda$, respectively, identifying characters in images is better than other methods. The Metric-Q and CPBD values for each approach are reported in Table II which validate our proposed method gives the highest quantitative measurement values. However, one can compare these methods in terms of time constraints in real-time applications.

\section{CONCLUSION}

In this study, an adaptive algorithm is proposed to address data error term selection and regularization parameter estimation in an image SR reconstruction. At first, in the data error counterpart, we have employed a simple but efficient weighting factor which incorporates less erroneous LR frames in the SR reconstruction. Then, based on BTV regularization, we have formulated the problem as finding the regularization parameter which satisfies the Morozov's discrepancy principle. Selecting the frames with lower error in data error term, efficient method for choosing the $\lambda$ (direct solving without using auxiliary variables), higher algorithm speed in $\lambda$ selection (fewer number of iterations than other automatic selection methods of $\lambda$ ) are among the achievements of this study. We have applied several experiments on some synthesized and real LR frames. Both visual and quantitative evaluation results show that the proposed algorithm can provide more appealing results than the other compared methods.

\section{REFERENCES}

[1] S. C. Park, M. K. Park, M. G. Kang, "Super-resolution image reconstruction: a technical overview," IEEE Signal Process. Mag., vol. 20, no. 3, pp. 21-36, May 2003. doi:10.1109/MSP.2003.1203207

[2] M. N. Bareja, C. K. Modi, "An improved iterative back projection based single image super resolution approach," Int. J. Image Graph., vol. 14, no. 04, p. 1450015, Oct. 2014. doi: $10.1142 / \mathrm{S} 0219467814500156$

[3] T. Lukes, K. Fliegel, M. Klima, "Objective image quality assessment of multiframe super-resolution methods," in Proc. 23rd International Conference Radioelektronika, Czech Republic, 2013, pp. 267-272. doi:10.1109/RadioElek.2013.6530929

[4] T. Wang, L. Cao, W. Yang, Q. Feng, W. Chen, Y. Zhang, "Adaptive patch-based POCS approach for super resolution reconstruction of 4D-CT lung data," Phys. Med. Biol., vol. 60, no. 15, pp. 5939-5954, Aug. 2015. doi:10.1088/0031-9155/60/15/5939 
[5] P. Vandewalle, L. Sbaiz, J. Vandewalle, M. Vetterli, "Superresolution from unregistered and totally aliased signals using subspace methods," IEEE Trans. Signal Process., vol. 55, no. 7, pp. 3687-3703, Jul. 2007. doi:10.1109/TSP.2007.894257

[6] M. K. Ng, A. C. Yau, "Super-resolution image restoration from blurred low-resolution images," J. Math. Imaging Vis., vol. 23, no. 3, pp. 367-378, Nov. 2005. doi:10.1007/s10851-005-2028-5

[7] M. Shen, C. Wang, P. Xue, W. Lin, "Performance of reconstructionbased super-resolution with regularization," J. Vis. Commun. Image Represent., vol. 21, no. 7, pp. 640-650, 2010 doi:10.1016/j.jvcir.2010.04.003

[8] L. T. Shao, H. G. Zhang, G. H. Zhang, "The improved hybrid MAPPOCS based algorithm for super-resolution image restoration research," Appl. Mech. Mater., vol. 701-702, pp. 373-380, Dec. 2014. doi:10.4028/www.scientific.net/AMM.701-702.373

[9] S. D. Babacan, R. Molina, A. K. Katsaggelos, "Parameter estimation in TV image restoration using variational distribution approximation," IEEE Trans. Image Process., vol. 17, no. 3, pp. 326-339, Mar. 2008 doi:10.1109/TIP.2010.2080278

[10] P. Milanfar, "Super-Resolution Imaging”, pp. 9-23,CRC Press, 2011.

[11] V. Patanavijit, S. Jitapunkul, "A robust iterative multiframe superresolution reconstruction using a Huber bayesian approach with Huber-Tikhonov regularization," in Proc. 2006 International Symposium on Intelligent Signal Processing and Communications, Yonago, Japan, 2006, pp. 13-16. doi:10.1109/ISPACS.2006.364825

[12] V. Patanavijit, S. Jitapunkul, “A robust iterative multiframe superresolution reconstruction using a bayesian approach with Tukey's biweight," in Proc. 2006 8th international Conference on Signal Processing, Beijing, China, 2006. doi:10.1109/ICOSP.2006.345547

[13] V. Patanavijit, S. Jitapunkul, "A lorentzian stochastic estimation for a robust iterative multiframe super-resolution reconstruction with Lorentzian-Tikhonov regularization," EURASIP J. Adv. Signal Process., vol. 2007, no. 1, p. 034821, 2007. doi:10.1155/2007/34821

[14] A. A. Hefnawy, "An efficient super-resolution approach for obtaining isotropic 3-D imaging using 2-D multi-slice MRI," Egypt. Informatics J., vol. 14, no. 2, pp. 117-123, 2013. doi:10.1016/j.eij.2013.03.003

[15] Q. Yuan, L. Zhang, H. Shen, "Regional spatially adaptive total variation super-resolution with spatial information filtering and clustering," IEEE Trans. Image Process., vol. 22, no. 6, pp. 2327 2342, Jun. 2013. doi:10.1109/TIP.2013.2251648

[16] S. Farsiu, M. D. Robinson, M. Elad, P. Milanfar, "Fast and robus multiframe super resolution," IEEE Trans. Image Process., vol. 13, no. 10, pp. 1327-1344, Oct. 2004. doi:10.1109/TIP.2004.834669

[17] V. Moram, S. D. Cabrera, "Superresolution using the optimal recovery framework with automatic generalized cross-validation," in Proc. Digital Signal Processing and Signal Processing Education Meeting (DSP/SPE), USA, 2011, pp. 344-349. doi:10.1109/DSPSPE.2011.5739237

[18] H. Z. Wang, S. Zhao, H.W. Lv, "Super-resolution image restoration with L-Curve," in Proc. 2008 Congress on Image and Signal Processing, China, 2008, pp. 597-601. doi:10.1109/CISP.2008.444

[19] Q. Yuan, L. Zhang, H. Shen, P. Li, “Adaptive multiple-frame image super-resolution based on U-curve," IEEE Trans. Image Process., vol 19, no. 12, pp. 3157-3170, Dec. 2010. doi:10.1109/TIP.2010.2055571

[20] V. A. Morozov, "Methods for Solving Incorrectly Posed Problems", pp. 32-153, Springer Press, New York, 1984. doi:10.1007/978-14612-5280-1

[21] D. Krawczyk-Stańdo, M. Rudnicki, "Regularization parameter selection in discrete ill-posed problems - the use of the U-Curve," Int
J. Appl. Math. Comput. Sci., vol. 17, no. 2, pp. 157-164, Jan. 2007. doi:10.2478/v10006-007-0014-3

[22] Y. W. Wen, R. H. Chan, "Parameter selection for total-variationbased image restoration using discrepancy principle," IEEE Trans. Image Process., vol. 21, no. 4, pp. 1770-1781, Apr. 2012 doi:10.1109/TIP.2011.2181401

[23] C. He, C. Hu, W. Zhang, B. Shi, "A fast adaptive parameter estimation for total variation image restoration," IEEE Trans. Image Process., vol. 23, no. 12, pp. 4954-4967, Dec. 2014 doi:10.1109/TIP.2014.2360133

[24] M. V Afonso, J. M. Bioucas-Dias, M. A. T. Figueiredo, "An augmented lagrangian approach to the constrained optimization formulation of imaging inverse problems," IEEE Trans. Image Process., vol. 20, no. 3, pp. 681-695, Mar. 2011. doi:10.1109/TIP.2010.2076294

[25] P. Purkait, B. Chanda, "Super resolution image reconstruction through bregman iteration using morphologic regularization," IEEE Trans. Image Process., vol. 21, no. 9, pp. 4029-4039, Sep. 2012 doi:10.1109/TIP.2012.2201492

[26] A. Panagiotopoulou, V. Anastassopoulos, "Regularized superresolution image reconstruction employing robust error norms," Opt. Eng., vol. 48, no. 11, p. 117004, Nov. 2009. doi:10.1117/1.3265543

[27] X. Zeng, L. Yang, "A robust multiframe super-resolution algorithm based on half-quadratic estimation with modified BTV regularization,” Digit. Signal Process., vol. 23, no. 1, pp. 98-109, Jan. 2013. doi:10.1016/j.dsp.2012.06.013

[28] H. Song, L. Qing, Y. Wu, X. He, "Adaptive regularization-based space-time super-resolution reconstruction," Signal Process. Image Commun., vol. 28, no. 7, pp. 763-778, Aug. 2013 doi:10.1016/j.image.2013.03.008

[29] J. F. Aujol, G. Gilboa, "Constrained and SNR-based solutions for TVHilbert space image denoising," J. Math. Imaging Vis., vol. 26, no. 12, pp. 217-237, Nov. 2006. doi:10.1007/s10851-006-7801-6

[30] A. Panagiotopoulou, V. Anastassopoulos, "Super-resolution image reconstruction techniques: trade-offs between the data-fidelity and regularization terms," Inf. Fusion, vol. 13, no. 3, pp. 185-195, Jul. 2012. doi:10.1016/j.inffus.2010.11.005

[31] B. Setiyono, M. Hariadi, M. H. Purnomo, "Survey of super-resolution using phased based image matching," Journal of Theoretical and Applied Information Technology, Vol. 43, pp. 245-253, Sep. 2012.

[32] J. Immerkær, "Fast noise variance estimation," Comput. Vis. Image Underst., vol. 64, no. 2, pp. 300-302, Sep. 1996. doi:10.1006/cviu. 1996.0060

[33] Z. Zheng Liu, R. Laganiere, "On the use of phase congruency to evaluate image similarity," in Proc. 2006 IEEE International Conference on Acoustics Speech and Signal Processing Proceedings, France, 2006, pp. II-937-II-940. doi:10.1109/ICASSP.2006.1660498

[34] Z. Wang, A. C. Bovik, H. R. Sheikh, E. P. Simoncelli, "Image quality assessment: from error visibility to structural similarity," IEEE Trans. Image Process., vol. 13, no. 4, pp. 600-612, Apr. 2004 doi:10.1109/TIP.2003.819861

[35] X. Zhu, P. Milanfar, "Automatic parameter selection for denoising algorithms using a no-reference measure of image content," IEEE Trans. Image Process., vol. 19, no. 12, pp. 3116-32, Dec. 2010. doi:10.1109/TIP.2010.2052820

[36] N. D. Narvekar, L. J. Karam, "A no-reference image blur metric based on the cumulative probability of blur detection (CPBD)," IEEE Trans. Image Process., vol. 20, no. 9, pp. 2678-2683, Sep. 2011 doi:10.1109/TIP.2011.2131660 